\title{
HIGH-RESOLUTION FUNCTIONAL PHOTOACOUSTIC TOMOGRAPHY
}

\author{
Lihong V. Wang*, Xueding Wang, Geng Ku, George Stoica \\ Optical Imaging Laboratory, Department of Biomedical Engineering \\ Texas A\&M University, 3120 TAMU, College Station, Texas 77843-3120 \\ URL: http://oilab.tamu.edu; Email: LWang@tamu.edu \\ * Corresponding author.
}

\begin{abstract}
All-optical imaging has high contrast but poor spatial resolution beyond the ballistic and quasiballistic regimes. All-ultrasonic imaging has high spatial resolution but poor contrast for early stage tumors. Photoacoustic tomography combines the high optical contrast and the high ultrasonic resolution. Our work in this emerging area of research will be summarized in this invited talk. In this technology, a diffraction-based inverse-source problem is solved in the image reconstruction, for which our group developed the rigorous reconstruction theory. We developed a prototype and accomplished non-invasive transdermal and transcranial functional imaging of small-animal brains in vivo.
\end{abstract}

Keywords - Photoacoustic tomography, ultrasonic resolution, optical contrast, inverse-source reconstruction

\section{INTRODUCTION}

Photoacoustic tomography (PAT, also called optoacoustic or thermoacoustic tomography) involves both photons and ultrasound. A short-pulsed laser source is used to irradiate the biological tissue samples under investigation. A temperature rise of the order of $\mathrm{mK}$ will be produced in a short time frame. Consequently, thermoelastic expansion will cause emission of acoustic waves, referred to as photoacoustic waves. The photoacoustic waves are measured by wideband ultrasonic transducers around the sample, and the acquired photoacoustic waves are used to reconstruct the optical absorption distributions. Because the laser pulse is short, proportionately high frequency ultrasonic waves will be produced and used to provide diffraction-limited spatial resolution.

Based on the above principle, the contrast in PAT is determined primarily by the optical properties of the biological tissues, and the spatial resolution in PAT is determined primarily by the photoacoustic waves originating from within the biological tissues. Photoacoustic tomography thereby combines the high optical contrast and the high ultrasonic resolution. This point is summarized in Table 1 .
Table 1. Motivation for PAT.

\begin{tabular}{|c|c|c|c|}
\hline & $\begin{array}{l}\text { All-optical } \\
\text { imaging } \\
\text { (Non- } \\
\text { ballistic) }\end{array}$ & $\begin{array}{l}\text { All-ultrasonic } \\
\text { imaging }\end{array}$ & $P A T$ \\
\hline Contrast & Excellent & $\begin{array}{l}\text { Poor for early } \\
\text { cancers }\end{array}$ & Excellent \\
\hline Resolution & Poor & $\begin{array}{l}\text { Excellent \& } \\
\text { scalable }\end{array}$ & Excellent \\
\hline Depth & Excellent & $\begin{array}{l}\text { Good \& } \\
\text { scalable }\end{array}$ & $\begin{array}{l}\text { Good \& } \\
\text { scalable }\end{array}$ \\
\hline $\begin{array}{l}\text { Speckle } \\
\text { artifacts }\end{array}$ & None & Strong & None \\
\hline Scattering & Strong & Weak & \\
\hline
\end{tabular}

II. FUNDAMENTALS OF PHOTOACOUSTICS: THE FORWARD PROBLEM

The basic equations in photoacoustic theory will be summarized here. If the electromagnetic pumping pulse duration is much shorter than the thermal diffusion time, thermal diffusion can be neglected; this is known as the assumption of thermal confinement. In this case, the acoustic wave $p(\mathbf{r}, \bar{t})$ is related to electromagnetic absorption, $H(\mathbf{r}, \bar{t})$, by the following wave equation:

$$
\frac{\partial^{2} p(\mathbf{r}, \bar{t})}{\partial \bar{t}^{2}}-\nabla^{2} p(\mathbf{r}, \bar{t})=\frac{\beta v_{s}}{C} \frac{\partial H(\mathbf{r}, \bar{t})}{\partial \bar{t}},
$$

where $\bar{t}=t v_{s} ; v_{s}$ is the acoustic speed, assumed to be constant; $C$ is the specific heat; and $\beta$ is the coefficient of volume thermal expansion. Eq. (1) can be rewritten in terms of $H(\mathbf{r}, \bar{t})$ :

$$
p(\mathbf{r}, \bar{t})=\frac{\beta v_{s}}{4 \pi C} \iiint \frac{\partial H\left(\mathbf{r}^{\prime}, t^{\prime}\right)}{\partial t^{\prime}} \frac{d \mathbf{r}^{\prime}}{\left|\mathbf{r}-\mathbf{r}^{\prime}\right|},
$$

where $t^{\prime}=\bar{t}-\left|\mathbf{r}-\mathbf{r}^{\prime}\right|$. The source term $H(\mathbf{r}, \bar{t})$ can further be written as the product of a purely spatial and a purely temporal component, i.e.:

$$
H(\mathbf{r}, \bar{t})=I_{0} \varphi(\mathbf{r}) \eta(\bar{t}),
$$


where $I_{0}$ is a scaling factor proportional to the incident radiation intensity; $\varphi(\mathbf{r})$ describes the electromagnetic absorption properties of the medium at $\mathbf{r}$; and $\eta(\bar{t})$ describes the shape of the irradiating pulse. Substituting Eq. (3) into Eq. (2) results in

$$
p(\mathbf{r}, \bar{t})=\frac{I_{0} \beta v_{s}}{4 \pi C} \iiint \varphi\left(\mathbf{r}^{\prime}\right) \frac{d \eta\left(t^{\prime}\right)}{d t^{\prime}} \frac{d \mathbf{r}^{\prime}}{\left|\mathbf{r}-\mathbf{r}^{\prime}\right|} .
$$

This equation provides the solution to the forward problem-prediction of the pressure outside the tissue if the absorption properties of the medium and the profile of the electromagnetic pulse are known. Its inverse problem needs to be solved for imaging.

\section{PHOTOACOUSTIC RECONSTRUCTION: THE INVERSE PROBLEM}

Our group derived the exact inverse solutions in planar, spherical, and cylindrical geometries [1-3]. These exact solutions are computationally intensive and were approximated to more efficient solutions in practical cases [4]. Usually, the distance between the photoacoustic sources and the detector is much longer than the wavelengths of the high-frequency photoacoustic waves that are useful for imaging. Under this far-field condition, the following approximate inverse solution holds:

$$
\varphi(\mathbf{r})=\left.C \iint_{S_{0}} d S_{0} \cos \left(\theta_{d}\right) \frac{1}{t} \frac{\partial p\left(\mathbf{r}_{0}, t\right)}{\partial t}\right|_{t=\left|\mathbf{r}_{0}-\mathbf{r}\right| / v_{s}},
$$

where $C$ is a constant; $S_{0}$ is the surface of detection; and $\theta_{d}$ is the angle between the normal of $d S_{0}$ and $\mathbf{r}-\mathbf{r}_{0}$ (the vector pointing from a point of detection to a point of reconstruction). It should be noted that Eq. (5) is a modified back-projection of quantity $\frac{1}{t} \frac{\partial p\left(\mathbf{r}_{0}, t\right)}{\partial t}$.

This back-projection is analogous to that in X-ray computed tomography $(\mathrm{CT})$. In $\mathrm{x}$-ray $\mathrm{CT}$, the backprojection is along the paths of the x-ray propagation. In PAT, the back-projection however is along the spherical shells that are centered at each of the ultrasonic detectors and that have a radius determined by the acoustic time of flight.

\section{EXPERIMENTS}

A configuration of the key components in our PAT system is shown in Fig. 1. An Nd:YAG laser was used to provide $532-\mathrm{nm}$ laser pulses with a width of $6.5 \mathrm{~ns}$. The laser beam was expanded and homogenized to provide an incident energy density of $<10 \mathrm{~mJ} / \mathrm{cm}^{2}$ on the skin of the rat head, which induced a temperature rise in the skin estimated to be $<20 \mathrm{mK}$. The photoacoustic waves were coupled into an ultrasonic transducer and were acquired around the head by scanning the ultrasonic transducer around the animal head in the horizontal imaging plane. The initial system required the immersion of the animal in the water tank for ultrasonic coupling, where the animal breathed through a tube. An improved system placed the animal below the water tank and coupled the ultrasonic signals through a deformable membrane at the bottom of the tank. When an ultrasound array becomes available to this technology, conventional gel coupling will be used to avoid the use of water coupling.

We used our PAT system to image the functional response of the cerebral cortex of rats to changes from hyperoxia to hypoxia. Non-invasive PAT images of the brain were taken when the animal inspired gas at $100 \% \mathrm{O}_{2}$ and $5 \% \mathrm{O}_{2}$, respectively. Subtracting the two PAT images produced a map corresponding to the functional changes of the cerebral cortex (Fig. 2) [5].

\section{SUMMARY}

We developed rigorous reconstruction algorithms, the foundation for PAT. We accomplished non-invasive transdermal and transcranial imaging of small-animal brains in vivo showing both structures and functions. Imaged structures include primarily the vasculature. Imaged functions include hemodynamic responses to various stimuli such as whisker stimulations, hyperoxia, and hypoxia.

\section{ACKNOWLEDGEMENTS}

This project was sponsored in part by the U.S. Army Medical Research and Materiel Command Grant No. DAMD17-00-1-0455, the National Institutes of Health Grants No. R01 EB000712 and No. R01 NS46214, and Texas Higher Education Coordinating Board Grant No. ARP 000512-0063-2001.

\section{REFERENCES}

1. M. Xu and L.-H. Wang, "Time-domain reconstruction for thermoacoustic tomography in a spherical geometry," IEEE Transactions on Medical Imaging 21 (7), 814-822 (2002).

2. Y. Xu, D. Feng, and L.-H. Wang, "Exact frequency-domain reconstruction for thermoacoustic tomography-I: Planar geometry," IEEE Transactions on Medical Imaging 21 (7), 823-828 (2002).

3. Y. Xu, M. Xu, and L.-H. Wang, "Exact frequency-domain reconstruction for thermoacoustic tomography-II: Cylindrical geometry," IEEE Transactions on Medical Imaging 21 (7), 829-833 (2002).

4. M. Xu, Y. Xu, and L.-H. V. Wang, "Thermo-acoustic imaging in biological tissues: Time-domain reconstruction algorithms", in preparation (2003).

5. X. Wang, Y. Pang, G. Ku, X. Xie, G. Stoica, and L.-H. Wang, "Non-invasive laser-induced photoacoustic tomography for structural and functional imaging of the brain in vivo," Nature Biotechnology[0] 21 (7), 803-806 (2003). 


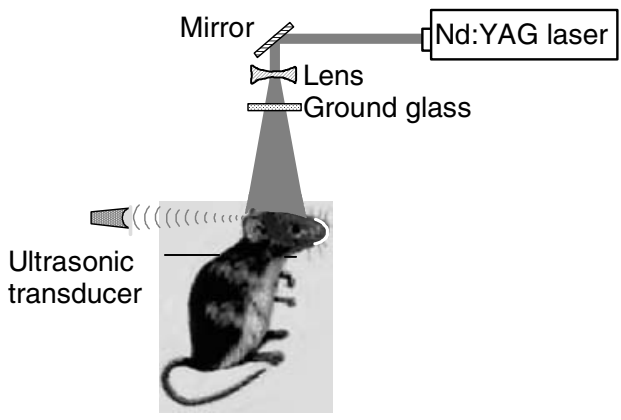

Fig. 1. Prototype PAT for non-invasive transdermal and transcranial imaging of the small-animal brain in vivo with the skin and skull intact.

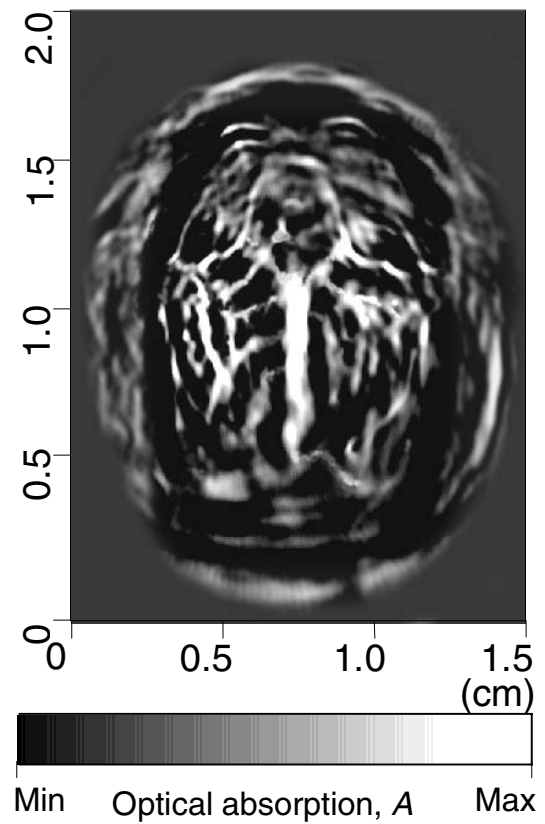

A

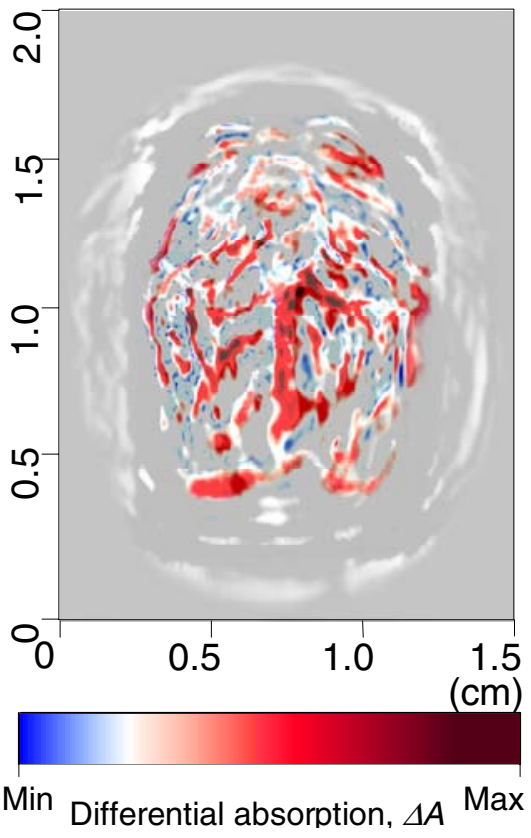

B

Fig. 2. Non-invasive photoacoustic tomography (PAT) of cerebral hemodynamic responses to the alteration from hyperoxia to hypoxia. (A) Non-invasive PAT image of vascular distribution in the rat cerebral cortex, which was acquired with the skin and skull intact. (B) Non-invasive functional PAT image of cerebral hemodynamic responses to altering the inspired gas from $100 \% \mathrm{O}_{2}$ to $5 \% \mathrm{O}_{2}$ acquired with the skin and skull intact. This functional map is superimposed on the image of the vascular distribution shown in (A). 